\title{
Acute Kidney Injury in Multisystem Inflammatory Syndrome in Children (MIS-C): a Case Report
}

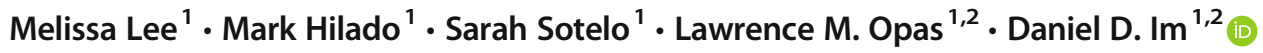

Accepted: 16 November 2020 / Published online: 21 November 2020

(C) Springer Nature Switzerland AG 2020

\begin{abstract}
A 15-year-old female with no significant past medical history who presented with abdominal pain, vomiting, and diarrhea, found to be in acute renal failure and was subsequently diagnosed with multisystem inflammatory syndrome in children (MIS-C). Critical manifestations seen in pediatric COVID-19 can be varied affecting different organ systems. Pediatric providers, during a pandemic with imperfect testing, must be keenly aware of how varied the pathogenesis of COVID-19 can be in children.
\end{abstract}

Keywords COVID-19 $\cdot$ MIS-C $\cdot$ Renal failure $\cdot$ AKI

$\begin{array}{ll}\text { Abbreviations } & \\ \text { PICU } & \text { Pediatric Intensive Care Unit } \\ \text { ARDS } & \text { Acute respiratory distress syndrome } \\ \text { MIS-C } & \begin{array}{l}\text { Multisystem inflammatory } \\ \text { syndrome in children }\end{array} \\ \text { COVID-19 } & \text { Coronavirus disease 2019 } \\ \text { SARS-CoV-2 } & \begin{array}{l}\text { Severe acute respiratory } \\ \text { syndrome coronavirus-2 } \\ \text { Bi-level positive airway pressure }\end{array}\end{array}$

\section{Introduction}

Severe acute respiratory syndrome coronavirus-2 (SARSCoV-2) has been found to cause a range of symptoms and disease states; however, the varied critical manifestations in children remain not well described. Most cases of SARSCoV-2 identified in children and adolescents have been asymptomatic, mild, or moderate, with only $2.1 \%$ being severe, and $1.2 \%$ being critical-with critical COVID-19 defined as children who can quickly progress to acute respiratory

This article is part of the Topical Collection on Covid-19

Daniel D. Im

danielim@usc.edu

1 Department of Pediatrics, LAC+USC Medical Center, 2020 Zonal Ave. IRD\#114, Los Angeles, CA 90033, USA

2 Keck School of Medicine, University of Southern California, Los Angeles, CA, USA distress or respiratory failure, and may also have shock, encephalopathy or heart failure, coagulation dysfunction, and acute kidney injury, including multiple organ dysfunction [1]. The mortality rate in children and adolescents is reported at 0 to $0.09 \%[1,2]$. In children and adolescents who are symptomatic, the most frequent symptoms are fever, cough, and diarrhea [2]. In one systematic review of literature reporting pediatric SARS-CoV-2 infection only $2 \%$ required PICU level care or mechanical ventilation [3].

A COVID-19 manifestation described that is unique to the pediatric population, multisystem inflammatory syndrome in children (MIS-C; also interchangeably known as pediatric inflammatory multisystem syndrome temporally associated with SARS-CoV-2 or PIM-TS) is a post-COVID infectious multisystem inflammatory state that can develop in children and holds clinical and laboratory similarities with other pediatric inflammatory diseases such as Kawasaki disease and toxic shock syndrome. The pathophysiology is still unclear, and clinical features are variable. The Centers for Disease Control case definition specifies that the patient be $<21$ years old, have "fever, laboratory evidence of inflammation, and evidence of clinically severe illness requiring hospitalization, with multisystem $(>2)$ organ involvement," in the absence of an "alternative plausible diagnosis," and evidence of SARS-CoV-2 infection or exposure [4].

\section{Case Presentation}

A 15-year-old female with no significant past medical history presented with 1 week of abdominal pain. The day prior to 
admission, she had two episodes of non-bloody and nonbilious emesis and two episodes of diarrhea. She had decreased oral intake the day of admission and subsequently experienced an episode of dizziness, which prompted the mother to call 911. Emergency Medical Services noted the patient to be dehydrated and gave her a 1-L normal saline bolus on route to the hospital. Upon arrival to the emergency department (ED), the patient was found to be febrile $\left(38.3^{\circ} \mathrm{C}\right)$, tachycardic (133 beats/min), hypotensive $(99 / 53 \mathrm{mmHg})$, and saturating $100 \%$ on room air breathing 20 breaths $/ \mathrm{min}$. She was noted to be alert and oriented with dry mucous membranes. She had a benign abdominal exam and was nonfocal for any areas of overt infection or trauma. She was found to have an erythematous, patchy, nonconfluent, and macular rash over the palms bilaterally, right wrist which spared the soles, and a similar oval-shaped rash on the sacral area. The patient denied any recent illnesses, fevers, cough, or upper respiratory tract infectious symptoms. The patient's mother verbalized that she was worried that the patient was not urinating because she was so dehydrated. The family had been staying at home self-isolating secondary to the ongoing pandemic. While they deny any known COVID-19 positive patients, the household was large, with the patient being one of multiple children within the household. Initial labs drawn in the ED were significant for a BUN of $54 \mathrm{mg} / \mathrm{dL}$ and creatinine of $5.78 \mathrm{mg} / \mathrm{dL}$. CBC was notable for bandemia. Labs were also notable for hyponatremia, hypokalemia, and elevations in CRP, procalcitonin, AST, ALT, INR, creatine kinase, probrain natriuretic peptide (pro-BNP), and troponin levels (Table 1). The initial COVID nasopharyngeal swab that was performed in the ED was negative. She was given a 1-L bolus of normal saline and was subsequently admitted to the PICU for management of her renal failure.

Initial differential diagnosis for this adolescent female presenting with fever, nausea, vomiting, diarrhea, and multiorgan involvement with impressive renal failure included the following: septic shock, toxic shock syndrome, MIS-C (despite the initial negative COVID swab results, antibodies were pending), typhoid fever, and rickettsial infection. The patient was initially started on clindamycin, ceftriaxone, and doxycycline. The patient's significant elevation of BUN and creatinine on the patient's labs indicated that the patient was in renal failure. The patient did not have any previously known history of renal disorders, or other medical issues that would indicate

Table 1 Initial lab results

\begin{tabular}{|c|c|c|c|c|c|}
\hline & Patient's values & Reference range & & Patient's values & Reference range \\
\hline Sodium (mmol/L) & 128 & $135-145$ & WBC (K/cumm) & 9.1 & $4.1-11.0$ \\
\hline Potassium (mmol/L) & 3.2 & $3.5-5.1$ & RBC (M/cumm) & 4.57 & $4.10-5.20$ \\
\hline Chloride $(\mathrm{mmol} / \mathrm{L})$ & 93 & $100-110$ & $\mathrm{Hgb}(\mathrm{g} / \mathrm{dL})$ & 11.3 & $12.2-14.8$ \\
\hline $\mathrm{CO} 2(\mathrm{mmol} / \mathrm{L})$ & 15 & $20-30$ & $\operatorname{Hct}(\%)$ & 33.60 & $36.3-43.4$ \\
\hline $\operatorname{AGAP}(\mathrm{mmol} / \mathrm{L})$ & 23 & $10-20$ & MCV (fL) & 73.5 & $81.4-95.0$ \\
\hline BUN (mg/dL) & 54 & $7-18$ & $\mathrm{MCH}(\mathrm{pg})$ & 24.7 & $24.5-32.6$ \\
\hline Creatinine (mg/dL) & 5.78 & $0.50-1.30$ & $\mathrm{MCHC}(\mathrm{g} / \mathrm{dL})$ & 33.6 & $32.3-35.3$ \\
\hline Glucose $(\mathrm{mg} / \mathrm{dL})$ & 131 & $65-99$ & $\mathrm{RDW}(\%)$ & 16.40 & $11.2-13.5$ \\
\hline Calcium (mg/dL) & 8.3 & $8.5-10.3$ & Platelets (K/cumm) & Clumped & $150-450$ \\
\hline Albumin (g/dL) & 4.1 & $3.5-5.0$ & Neutrophil Manual (\%) & 80 & - \\
\hline Alk Phos (U/L) & 70 & $116-468$ & Band Manual (\%) & 15 & - \\
\hline AST (U/L) & 51 & $10-50$ & Lymph Manual (\%) & 1 & - \\
\hline ALT (U/L) & 47 & $10-50$ & Monocyte Manual (\%) & 2 & - \\
\hline Bili total (mg/dL) & 0.5 & $<1.0$ & Eos Manual (\%) & 2 & - \\
\hline Bili direct (mg/dL) & 0.2 & $<0.3$ & Abs Neut Man (K/cumm) & 8.6 & $1.8-8.0$ \\
\hline Protein total $(\mathrm{g} / \mathrm{dL})$ & 7.2 & $6.0-8.0$ & Abs Lymph Man (K/cumm) & 0.1 & $1.5-5.2$ \\
\hline $\mathrm{CK}(\mathrm{U} / \mathrm{L})$ & 1195 & $20-180$ & Abs Monocyte Man (K/cumm) & 0.2 & $0.0-0.8$ \\
\hline Troponin $(\mathrm{ng} / \mathrm{mL})$ & 0.08 & $<0.00$ & Abs Eos Man (K/cumm) & 0.2 & $0.0-0.5$ \\
\hline Pro BNP NT (pg/mL) & 4655 & $<125$ & Platelet estimate (K/cumm) & $150-300$ & - \\
\hline PT (s) & 15.4 & $11.8-14.4$ & & & \\
\hline INR & 1.23 & $0.87-1.13$ & & & \\
\hline PTT (s) & 19.9 & $24.4-36.6$ & & & \\
\hline Fibrinogen (mg/dL) & 812 & $237-481$ & & & \\
\hline D-Dimer $(\mathrm{mcg} / \mathrm{mL})$ & 6.00 & $<0.49$ & & & \\
\hline
\end{tabular}


previous renal insult. The initial constellation of labs was inconsistent with a chronic kidney disease, and more consistent with pre-renal acute kidney injury and acute tubular necrosis. Given the severity of laboratory findings, possibility of needing renal replacement therapy was discussed, but deferred in the setting of the patient's significant dehydration. Judicious rehydration therapy with intravenous fluids was initiated with the serious concerns of the elevated cardiac markers potentially suggesting a concomitant heart failure. However, further cardiac work-up revealed that, despite the elevated troponin values, the patient's EKG did not show any evidence of cardiac ischemia, no other evidence of cardiac involvement or failure. Given the concern for heart failure and involvement of coronary arteries in MIS-C, echocardiogram was performed. Echocardiography revealed normal anatomy, normal coronaries, and a normal shortening fraction of $30 \%$.

By hospital day 2, given the mounting concern for MIS-C and no clear source of infection in the setting of persistent fevers $\left(\mathrm{T}_{\max } 40.3^{\circ} \mathrm{C}\right)$, elevated inflammatory markers despite broad antibiotic coverage, the patient was empirically given intravenous immunoglobulin (IVIG) at a dose of $2 \mathrm{~g} / \mathrm{kg}$. COVID antibodies (IgG, IgM) returned positive on hospital day 3 which confirmed the diagnosis of MIS-C. With all other cultures negative, antibiotics were discontinued. A dose of intravenous methylprednisolone given concern for IVIG treatment resistance. The patient remained persistently oliguric on hospital days 1 to 3 with a peak BUN and Cr of $86 \mathrm{mg} / \mathrm{dL}$ and $7.67 \mathrm{mg} / \mathrm{dL}$, respectively, on hospital day 4 . With a reassuring evaluation of cardiac function, rehydration with intravenous fluids was continued and the utilization of diuretic therapy increased the patient's urine output. Starting hospital day 5, the patient began to show persistent improvement in overall clinical status after completion of IVIG and methylprednisolone with down trending fevers, inflammatory markers, and improving renal function.

The patient continued to do well throughout the rest hospital course and was discharged on hospital day 6 . Repeat echo completed prior to discharge showed shortening fraction between 28 and 32\%, no ectasias or aneurysmal changes of coronaries. The patient was discharged with aspirin for prophylaxis given risk for hypercoagulability secondary to MISC. Four weeks following discharge, the patient was seen in a follow-up visit and found to be doing well, with her BUN and $\mathrm{Cr}$ found to be $5 \mathrm{mg} / \mathrm{dL}$ and $0.77 \mathrm{mg} / \mathrm{dL}$ respectively.

\section{Discussion}

Critical pediatric COVID-19 is uncommon compared to the critical manifestations in adult COVID-19, but we are learning that it is just as heterogenous, albeit in a different manner. Not only can a child present with acute hypoxemia and ARDS due to COVID-19, a child can also present with severe renal failure due to MIS-C. To our knowledge, this is the first case report detailing renal failure in MIS-C.

Acute kidney injury has a reported worldwide incidence of $8.9 \%$ in adult COVID-19 patients [5]. Autopsy studies show that some patients had renal biopsy evidence of kidney injury even though they did not have associated lab abnormalities. This suggests that acute kidney injury (AKI) may be even more prevalent in COVID-19 patients than currently reported. Less is known about the prevalence of AKI in pediatric COVID patients. Batlle et al. proposed a multifactorial mechanism of AKI in patients infected with SARS-CoV-2. AKI and renal failure may be due to a combination of direct infection of renal parenchyma through ACE- 2 proteins, and microvascular injury caused by a COVID-19 triggered cytokine storm, macrophage activation and hypercoagulability [6].

In existing pediatric literature on MIS-C, AKI has been reported in 2 to $8 \%$ of children with MIS-C in the USA [7]. There are currently no published reports detailing specific presentations kidney failure in MIS-C. The proposed pathophysiology of MIS-C is strikingly similar to the proposed mechanism of AKI in COVID infections. The delayed presentation of MIS-C of up to a month after respiratory symptoms of COVID-19, and the elevated levels of pro-inflammatory cytokines suggest that MIS-C is caused by post-infectious immune dysregulation [8]. Although much of the discussion around MIS-C has centered on cardiac manifestations, pediatric providers must be cognizant of the renal manifestations of COVID-19. Interestingly, the patient in this case report had no clinically relevant cardiac manifestations and the elevated cardiac markers were likely secondary to accumulation secondary to renal failure [9].

We are faced with a new disease entity that behaves similarly to diseases we have treated before yet confounds our expectations. We are learning that this single organism can cause a wide spectrum of life-threatening disease, and we are still working to understand why. Children infected with SARS-CoV-2 can present as asymptomatic carriers, or with multisystem inflammatory syndrome and kidney failure, or with severe ARDS. This broad spectrum of possible presentations has made the diagnosis and management of COVID19 , and the prevention of outbreaks among pediatric populations particularly difficult. Only through continued global collaboration that we can continue to rapidly expand our understanding of SARS-CoV-2 infection in children, its course in pediatric critical COVID-19, and the best ways in which we can best treat our patients.

Authors' Contributions Drs. Lee, Hilado, Sotelo, Opas, and Im were all involved in conceptualizing and designing the case report. All authors were involved in the literature review and drafting of the manuscript. All authors approved the final manuscript as submitted and agree to be accountable for all aspects of the work.

Data Availability Not applicable. 


\section{Compliance with Ethical Standards}

Conflict of Interest The authors declare that they have no conflicts of interests.

Ethics Approval Not applicable.

\section{Consent to Participate Not applicable.}

Consent for Publication Informed consent for case report received from patient's guardians.

Code Availability Not applicable.

\section{References}

1. Souza TH, Nadal JA, Nogueira RJN, Pereira RM, Brandão MB. Clinical manifestations of children with COVID-19: a systematic review. Pediatr Pulmonol. 2020;55(8):1892-9.

2. Mantovani A, Rinaldi E, Zusi C, Beatrice G, Saccomani MD, Dalbeni A. Coronavirus disease 2019 (COVID-19) in children and/ or adolescents: a meta-analysis. Pediatr Res. Published online June 17, 2020.
3. Liguoro I, Pilotto C, Bonanni M, Ferrari M, Pusiol A, Nocerino A, et al. SARS-COV-2 infection in children and newborns: a systematic review. Eur J Pediatr. 2020;179(7):1029-46.

4. CDC. Multisystem Inflammatory Syndrome in Children (MIS-C). Centers for Disease Control and Prevention. Published February 11, 2020. https://www.cdc.gov/mis-c/hcp/. Accessed 9 Oct 2020.

5. Chen YT, Shao SC, Hsu CK, Wu IW, Hung MJ, Chen YC. Incidence of acute kidney injury in COVID-19 infection: a systematic review and meta-analysis. Crit Care. 2020;24(1):346.

6. Batlle D, Soler MJ, Sparks MA, Hiremath S, South AM, Welling PA, et al. Acute kidney injury in COVID-19: emerging evidence of a distinct pathophysiology. J Am Soc Nephrol. 2020;31(7):1380-3.

7. Feldstein LR, Rose EB, Horwitz SM, Collins JP, Newhams MM, Son MBF, et al. Multisystem inflammatory syndrome in U.S. children and adolescents. N Engl J Med. 2020;383(4):334-46.

8. Dufort EM, Koumans EH, Chow EJ, Rosenthal EM, Muse A, Rowlands J, et al. Multisystem inflammatory syndrome in children in New York state. N Engl J Med. 2020;383(4):347-58.

9. Savoj J, Becerra B, Kim JK, Fusaro M, Gallieni M, Lombardo D, et al. Utility of cardiac biomarkers in the setting of kidney disease. Nephron. 2019;141(4):227-35.

Publisher's Note Springer Nature remains neutral with regard to jurisdictional claims in published maps and institutional affiliations. 